\title{
An Overview of NASA's Intelligent Systems Program
}

\author{
Daniel E. Cooke \\ NASA Ames Research Center \\ Mountainview. CA \\ dcooke@mail.arc.nasa.gov \\ and \\ Texas Tech University \\ Lubbock. TX \\ dcooke@coe,rtu.edu
}

\begin{abstract}
Hostruct- NASA and the Computer Science Research community are poised to enter a critical era. An era in which - it seems - that each needs the other. Market forces, driven by the immediate economic viability of computer science research results, place Computer Science in a relatively novel position. These forces impact how research is done, and could, in worst case, drive the field away from significant innovation - opting instead for incremental advances that result in greater stability in the market place.

NASA, however, requires significant advances in computer science research in order to accomplish the exploration and science agenda it has set out for itself. NASA may indeed be poised to advance computer science research in this century. much the way it advanced aero-based research in the last.
\end{abstract}

\section{TABLE OF CONTENTS}

1. INTRODUCTION
2. AUTONOMOUS SYSTEMS
3. HUMAN CENTERED COMPUTING
4. INTELLIGENT DATA UNDERSTANDING
5. REVOLUTIONARY COMPUTING
6. RESEARCH MODEL FOR COMPUTER SCIENCE
7. CONCLUSIONS

\section{INTRODUCTION}

For NASA to attain its exploration and science objectives, it must advance computer science research. In fact NASA's most critical research needs are no longer in the realm of aero-based research, but are now in the realm of computer science. The Intelligent Systems Program is the first coherent national initiative focused on computer science.

There are three problems facing future NASA activities that motivate the research elements of the Intelligent Systems Program. The first has to do with the communication delays that face longer distance human and robotic missions of the future. These delays force future mission planners to face the reality that their missions will not be able to rely upon the ground-based remote control that has benefited past missions - whether the missions involve humans or robots. Greater autonomy for both types of missions must be a major characteristic of missions to Mars and beyond.
Given the lack of ground-based input. future autonomous systems for missions must accommodate uncertainty to an extent much greater than has been accommodated in the past - even beyond that attained in the remote agent experiment on Deep Space One. Whereas uncertain situations could default to humans on earth in the past, the distances involved with future missions reduce the reliance on earthbased remote controls by humans. Therefore, greater autonomy is required in future human and robotic missions. [1]

\section{ALTONOMOL'S SYSTEMS.}

The autonomous nature of past systems has been based upon a systematic approach where scientists attempted to predict every eventuality that might occur and programmed a response or action for the system to take should the event occur. If an event occurred that the system was not programmed to handle, earth-based controllers took over. These types of systems are not adequate for future missions. Given the uncertainties involved in exploration, conditionresponse systems are too fragile often rely too heavily on the ground-based safety nets.

One can view the programming of past systems like raising a child. For example, children can be instructed in the following way:

If someone hits you, do not hit back:

Under no circumstance should you start a fight:

Under no circumstance should you throw rocks at another; etc.

To raise a child in this manner is difficult. It is unlikely that every event or situation the child may encounter can be covered. Due to the uncertainty of future events, the child is likely to encounter a sination where the system of rules fails him or her. A better approach is to raise the child in manner where subsuming, guiding principles like:

\section{Treat others the way you wish to be treated.}

are accommodated. A child raised in a way that this type of principle can operate effectively will be better prepared to handle unforeseen situations. Likewise, autonomous 
iatems of the future must accommodate guiding principles. There are a number of promising approaches. model-based reasuning approaches (e.g. Bayesian Decision Models, Exiended Logic Programming. Neural Network. and Hybrid approaches). that will result in more robust autonomous systems that can deal much more effectively with the uncertainty one encounters in space and planetary exploration

\section{Human Centered Computing}

Autonomous systems will certainly affect NASA's ability to deploy robotic platforms into deeper space. They will also enable NASA's ability to deploy humans into long distance exploration missions. As an example mission, consider the human exploration of Mars. Because of the communication delays inherent in such a mission, astronauts will need to exercise greater autonomy - delegating a great deal of responsibility to computers that will need to be much more intelligent than the systems currently deployed.

Effectively, the Human-Centered Computing research is meant to result in systems that take into account the level of intelligence and capability of the systems deployed, together with the cognitive and perceptual abilities of the astronauts deployed - resulting in optimal systems of humans and machines where the machines do what they do best. freeing humans to do the more creative activities that they do best.

For example, a lot of mission operations currently performed by human controllers on earth will need to be automated so that the astronauts continue to receive much of their guidance from sources to which the scheduling and controlling can safely be delegated. Mission ops in a box among other capabilities - will be an important, enabling characteristic of future missions.

To give a further explanation of this revolutionary approach to systems design, consider past epochs of human experience. [2] In the agrarian society of the past, humans equaled physical labor. Since humans spent the majority of their time performing labor, they had very little time left to perform advanced problem solving, theory formulation, and the other more creative activities required for invention and discovery.

In the industrial society machines began performing physical labor and the humans equaled the brains of these machines. Humans were freer to perform advanced cognitive activities during this epoch, and science made great strides. In the information age the brain of the human is extended and enhanced by a machine - the computer. Even trivial applications significantly extend human capabilities. Knowledge and the application of knowledge are embodied in software. For example, many people now prepare their taxes with the help of software tools that possess much of the skill and knowledge possessed by tax experts. As the intelligence of systems increases, the more mundane and lower level reasoning activities can be delegated to computers. freeing the humans to perfurm the more advanced and creative cognitive activities. In tuture exploration missions. humans cannot be mired in the details of mission operations or even vehicle health and maintenance. Humans must be freed to perform the discovery objectives of the missions. Humans excel at putting seemingly disjoint concepts together - they types of cognitive activities that are at the heart of invention and discovery. Computers do not excel at these kinds of activities, but do excel and out perform humans when it comes to more routine and sometimes tedious mental activities.

Results in this area will affect not only human exploration of distant planets, but also the abilities of humans on earth, performing. e.g., mission operations, air traffic control, etc.

\section{Intelligent Data Understanding}

The second motivation for the IS program arises out of the problems NASA scientists and engineers face when trying to analyze and understand the vast amounts of vehicle maintenance data and data acquired through the use of earth and space observing instruments. Currently, NASA is receiving nearly two terabytes of data per day from earth observing satellites alone. NASA is able to acquire and store vast amounts of data, but the amount of data is duly stressing humankind's ability to analyze the data.

One can view these vast data sets as experimental or observed data. Empirical observations are typically reduced to concise theories, which explain the observations. In addition to data mining and knowledge discovery research, the Intelligent Data Understanding program element is intending to provide approaches that will better enable scientists to construct theories based upon the data acquired. Revolutionary approaches that provide theory-based access to these data sets are included in the goals of this program element.

Data sets at NASA are not always contained in a database. In fact, most of the data NASA acquires is contained in flat files that possess format information in their headers. Traditional approaches to data mining and knowledge discovery are, therefore, not always relevant to the needs of NASA.

A major result in this program element would be if these data sets could be reduced to much smaller algorithmic units. These algorithms could be viewed as concise statements of the data - providing more manageable representations of the data that should lead to betrer understanding - and perhaps might be capable of reproducing the data sets - thus resulting in much more significant data compression.

Fundamental results in this element should have wide application, providing new analytical tools to assist scientists in understanding space and earth science data, and engineers in understanding vehicle and instrument maintenance data. Clearly application to other types of data, 
e. internet databases. is a potential side effict of research In this area. Ultimately. the Intelligent Data Understanding program element is focused on the following set of transtiormations:

- Dala mining - changing lata to information.

- Knowledge Discovery - changing information to knowledge und theories

- Wachine Learning - hoving the machine trained to do mining and discovery with increasingly less humun intervention.

- tction - the knowledge gained should result in actions that improve our stewardship of the world's resources

\section{REvOlutionitry COMPUTING}

The third motivation for the program is the size, weight, energy consumption problems, and space hazards that beset space-based computations. The possibilities of quantum and molecular computing provide answers to some of NASA's concerns about computing in space.

The radiation and solar effects on computing can be offset by the massive parallelisms these approaches may offer. The size. weight, and power consumption concerns are also positively affected by these newer approaches to computing architectures. Perhaps the most important benefit is the new computational models and computer languages that may be implied by these approaches. Revolutionary computing approaches are radically different from the traditional von Neumann and even the more conventional non-von Neumann approaches to architecture. As such, the computational models implied may provide radically new insights into problem solving - even (possibly) helping scientists to find tractable solutions to problems for which only intractable algorithms are currently known. These algorithms may allow for feasible algorithms within the constraints of current technologies.

More straightforward solutions to problems may result. (Currently solutions to these problems are approximate solutions - due to the intractability of the problems making them much more complex to develop.) The revolutionary computing program element is focused not on building quantum or molecular computers, but upon the computational models and languages implied by these approaches, as well as in the development of specific NASArelevant algorithms that would allow for the immediate exploitation of these technologies if and when they become available.

\section{Research Model for Computer Science}

The computer science community is faced with market forces, which could ultimately impede its ability to perform state-of-the-art research. To combat the potential for stagnation, the research community has been in need of a major force to provide direction and leadership in some key areas. In particular, due to the aforementioned market forces, the theoretical computer science community has, for the most part, lacked a signiticant and organized experimental community. Without an experimental community it is difficult to chart progress and provide convincing evidence of the significance of a theoretical result. The is program has sought to advance the notion that hard application areas could be an excellent substitute for experiment. NASA has great hard applications

The program is also determining better ways to transition the fundamental results produced by the research efforts managed by the program to products. We believe that a different model of software engineering would help a great deal. A proof-of-concept arising from the theoretical community, tested against a hard application will not necessarily transition into practice.

To take an idea from proof-of-concept and actually fly it onboard an aircraft or a spacecraft is a major undertaking.

To address this issue, we believe that some elements of software engineering need redefinition or refinement. Theorists are not likely to take their idea all the way to product. This requires software engineers. We believe the notion of Joint Application Development should be expanded to include more than problem domain experts: to include the theorists who developed the approach or idea that is being taken to product. This is not a new idea. Years ago, Richard Feynman, while working at Los Alamos, was dispatched to Oakridge, where engineers were building the plants to produce the materials for the atom bomb. The engineers needed to be briefed on the theoretical aspects and context within which they were working. After the briefing, the engineers were able to correct serious problems in their initial designs and ultimately construct the plants that served a major role in winning World War II.

The program office has worked to advance this notion to the computer science community and inside NASA. The advantage for the computer science community is that NASA could become its experimental community. The advantage for NASA is that Computer Scientists begin training their research - even theoretical research - on NASA relevant problems.

\section{SUMMARY}

In [3] we reported the recent shift of research priorities at NASA. In the last four years Ames has shifted its focus from aero-based research to Computer Science Research. In the shadow of the largest wind tunnel in the world and in the heart of Silicon Valley, priority one is now Computer Science research. To achieve its future exploration and science agenda, NASA's most critical research needs are in the area of Intelligent Systems. NASA is now positioning itself to advance Computer Science research in this century in the same way that it advanced aero-based research in the last. 


\begin{abstract}
"Ames has been and is now develuping partnerships with academia and with Silicon Valley companies in order to abbreviate the time it takes to transition new developments into industrial practice and products. This etfort is meant to strengthen Ames' role in providing computer science capabilities for NASA's must stressing applications (e.g. spacecratt autonomy) and to establish an additional role for Ames .. to provide intrastructure for long term research that much of the computer industry can no longer undertake due to market pressures and the rapidity of new developments. As more and more of computer science becomes commodity it is important to establish forces that facilitate research. In this manner computer science can be market driven in practice and science driven in the development of the state of the art. "[3]
\end{abstract}

The Intelligent Systems program is to foster and develop the research landscape so that NASA can indeed begin advancing computer science research on a coherent and united front.

\title{
REFERENCES
}

[1] Intelligent Systams Program Plan, NASA Ames Research Center.

[2] Wojciech Cellary. "Knowledge and Soltware Engineering in the Global Infomation Sociay," Keynote Address, 2000 International Conference on Software Eng. and Knowledge Eng.. Chicago, IL, July 6-8,2000.

[3] Daniel E. Cooke and Scott Hamilton, "New Directions for NASA Ames Research Center," COMPUTER (January 2000) Vol. 33 No. l, pp.63-71.

DanielCooke, Ph.D. serves as the Manager of NASA's Intelligent Systems Program and as Professor and Chair of the Computer Science Department at Texas Tech University. Previously. Dr. Cooke served as an Assistant, Associate, and then Professor and Chair of the Computer Science Department at the University of Texas at El Paso (UTEP). 